\title{
Coupled dynamic model and vibration responses characteristic of a motor-driven flexible manipulator system
}

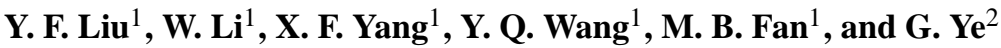 \\ ${ }^{1}$ School of Mechatronic Engineering, China University of Mining and Technology, Xuzhou, China \\ ${ }^{2}$ School of Mechanical \& Electrical Engineering, Jiangsu Normal University, Xuzhou, China
}

Correspondence to: W. Li (liweicumt@163.com)

Received: 2 November 2014 - Revised: 20 May 2015 - Accepted: 8 October 2015 - Published: 21 October 2015

\begin{abstract}
Motor-driven flexible manipulator systems (MDFMSs) are widely used in industry robot fields. During the dynamic modeling, the separate investigation method which neglects the dynamic behavior of the driving motor will cause an error in the dynamic analysis of the flexible manipulator, especially with high-speed operations. This paper proposes a coupled dynamic model of the MDFMS in which the driving motor and flexible manipulator are considered as an integrated system, which can clearly reflect the influence of the dynamic effect of the driving motor. Based on the proposed dynamic model, the vibration responses of the flexible manipulator under different velocities, accelerations and structure parameters, as well as the effect mechanism of the driving motor on the vibration responses, are investigated. The results obtained in this paper contribute to the structure design, motion optimization and dynamic analysis of flexible manipulators.
\end{abstract}

\section{Introduction}

Flexible manipulators have been actively developed and widely used, particularly in aerospace and robot fields because of their lightweight feature (Ge et al., 1998; Dwivedy and Eberhard, 2006), which can meet the purposes of highspeed operation and high energy utilization efficiency. Due to their constructional features, however, the damp and stiffness of the flexible manipulators are generally lower; as a result, undesirable elastic deformations and residual vibrations will be easily excited during the operation tasks of the flexible manipulators, such as starting, transforming gestures and stopping, which are inevitable and have a significant influence on the operation precision and structural life of precision operating systems, especially in high-speed operations (Dwivedy and Eberhard, 2006; Maria et al., 2013). In order to control the flexible manipulators more accurately, numerous researchers have studied the dynamic modeling of the flexible manipulators in order to investigate the dynamic behaviors (Mohsen et al., 2004; Qiu, 2012; Kerem et al., 2009; Qiu et al., 2012).
As a typical coupled system, the flexible manipulator can be modeled as a flexible arm and a driving base (Siciliano and Khatib, 2008). Generally, the flexible arm can be described as a flexible beam to reflect its lower damp and stiffness as well as its elasticity properties, while the driving base is defined by translational motions or rotational motions for driving the flexible arm and executing the tasks. In these existing studies, however, the dynamic investigations have mainly focused on the flexible arm and the dynamic behavior of the driving base has been neglected. Due to the fact that the flexible arm is coupled with the driving base, this separate investigation method will cause an error in the dynamic analysis (Zhang et al., 2010; Li et al., 2008). On the other hand, as a typical electromechanical integrated system, the motor-driven flexible manipulator system (MDFMS) conveys the driving motor, transmission mechanism and flexible arm and will exhibit complex dynamic behaviors because of the coupled effect between each component (Siciliano and Khatib, 2008), which will influence the performance of the flexible manipulator and lead to certain motion fluctuations in the driving force and torque or the motion velocities and accelerations of the driving base, especially for systems using harmonic 
drives (Zhao et al., 2014). The main reason for this is the effect of the motor parameters and mechanism inertias ( $\mathrm{Li}$ et al., 2008; Liou et al., 1991; Andreaus and Casini, 2001; Liu et al., 2015), such as the unbalanced mass of motor in different rotational speeds and the backlash between the splines (Tuttle and Seering, 1996). However, it is worth noting that the driving force and torque or the motion velocities and accelerations of the driving base in the existing studies are assumed to be constant without any fluctuations (Li et al., 2008; Wei et al., 2006). From the above analysis we can see that this ideal assumption deviates from the actual cases and will cause an error in the dynamic analysis for a precision system. Thus, in order to conduct an accurate dynamic analysis for the flexible manipulator, the dynamic modeling should also consider the dynamic behavior of the driving base. The observation by Smaili et al. (1996) indicated that their devised dynamic model, which treats the linkage and its drive train as a system, can provide a more accurate estimation for the dynamic response of a mechanism during start-up. Considering the driving motor and the linkage mechanism as an integrated system, Li et al. (2008) investigated the nonlinear vibration of a three-phase AC motor-linkage mechanism system.

From reviewing these current studies, it can be seen that most of the related investigations considering the dynamic effect of the driving motor mainly focus on the linkage mechanism systems and that few surveys have studied the flexible manipulator. Moreover, as the flexible manipulator has actual motions and structure parameters, the effect mechanism of the dynamic effect on the vibration responses characteristic of the flexible manipulator under different motions and structure parameters, as well as the strategies to suppress the influence of the driving motor, has not yet been reported. The objectives of this paper are to establish the coupled dynamic model of the MDFMS considering the dynamic effect of the driving motor and investigate the dynamic property and vibration responses characteristic of the flexible manipulator under different motion velocities, accelerations and structure parameters. Also, based on the proposed dynamic model and analysis, the effect mechanism of the dynamic effect of the driving motor on the vibration characteristic and the relevant suppressing strategies are presented, which is meaningful for the motion optimization and vibration control for the flexible manipulator. The remainder of this paper is organized as follows. Section 2 presents the coupled dynamic model of the MDFMS. The analysis results based on the dynamic model are presented and discussed in Sect. 3. Finally, Sect. 4 concludes the paper.

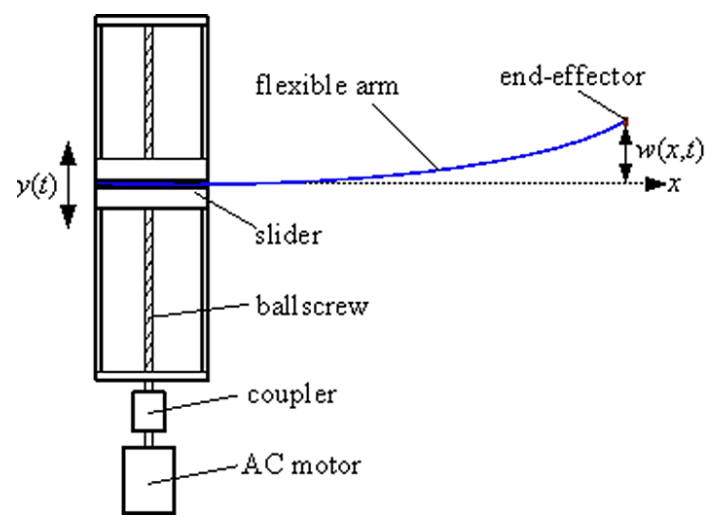

Figure 1. Schematic diagram of the MDFMS.

\section{Coupled dynamic model of the MDFMS}

A schematic diagram of the MDFMS is shown in Fig. 1, which gives a description of the comprising components: the driving motor connects the ball screw system through a coupler, and the flexible arm together with the end effector on its tip is clamped on the moving slider of the ball screw system. Due to the damp and stiffness being lower, the flexible arm and the end effector will be easily excited by elastic deformations and residual vibrations during the operation tasks. These undesirable elastic deformations and residual vibrations conflict with the precision demand of the end effector. In this section, to analyze the dynamic performance of the flexible manipulator, the coupled dynamic model taking the driving motor and the flexible arm as an integrated system is firstly established, and the dynamic equations obtained will be used for the following investigation of the characteristic vibration responses.

\subsection{Dynamic equation of the motor and flexible manipulator system}

As an integrated system, the dynamic equation of the MDFMS should consider the behaviors of the driving motor and the flexible arm. In the dynamic modeling, the flexible arm is modeled as a flexible beam to describe its lower damp and stiffness properties, and it is assumed that the flexible beam satisfies the Bernoulli-Euler beam assumption, and the transverse vibrations are the main motions considered. Thus, the transverse vibration displacement of the flexible manipulator can be expressed as

$Y(x, t)=y(t)+w(x, t)$,

where $y(t)$ denotes the displacement of the moving slider and $w(x, t)$ denotes the transverse vibration displacement of the flexible arm; according to the assumed modes method (Singiresu, 2004), this yields 
$w(x, t)=\sum_{i=1}^{n \rightarrow \infty} W_{i}(x) q_{i}(t)$,

where $q_{i}(t)$ denotes the $i$ th generalized coordinate and $W_{i}(x)$ denotes the $i$ th orthogonal mode shapes, which can be written as (Singiresu, 2004)

$W_{i}(x)=\sin k_{i} x-\sinh k_{i} x+\zeta\left(\cos k_{i} x-\cosh k_{i} x\right)$.

Here $\zeta=-\frac{\sin k_{i} L+\sinh k_{i} L}{\cos k_{i} L+\cosh k_{i} L}, k_{i}^{4}=\rho A \omega_{i}^{2} / E I$, in which $\omega_{i}$ is the $i$ th natural frequency of the flexible arm; $L, \rho, A$ and $E$ are the length, mass density, cross-sectional area and Young's modulus of the flexible arm, respectively; and $I$ denotes the cross-sectional moment of inertia about the neural axis and can be expressed as $I=b h^{3} / 12$, where $b$ and $h$ are the width and thickness of the flexible arm, respectively.

The kinetic energy of the MDFMS is

$$
\begin{aligned}
E_{k} & =\frac{1}{2} m_{\mathrm{s}}\left(\frac{\mathrm{d} y}{\mathrm{~d} t}\right)^{2}+\frac{1}{2} m_{\mathrm{t}}\left[\frac{\mathrm{d} y}{\mathrm{~d} t}+\frac{\partial w(L, t)}{\partial t}\right]^{2} \\
& +\frac{1}{2} \int_{0}^{L} \rho A\left(\frac{\mathrm{d} y}{\mathrm{~d} t}+\frac{\partial w}{\partial t}\right)^{2} \mathrm{~d} x .
\end{aligned}
$$

Here, the first part of the equation denotes the kinetic energy of the moving slider, the second part denotes the kinetic energy of the end effector, and the third part denotes the kinetic energy of the flexible arm; $m_{\mathrm{s}}$ and $m_{\mathrm{t}}$ are the mass of the moving slider and the end effector, respectively.

The potential energy of the MDFMS mainly considers the elastic potential energy of the flexible arm and can be expressed as

$$
E_{\mathrm{p}}=\frac{1}{2} \int_{0}^{L} E I\left[\frac{\partial^{2} w(x, t)}{\partial x^{2}}\right]^{2} \mathrm{~d} x .
$$

According to Eq. (2), Eqs. (4) and (5) can be further written as

$$
\begin{aligned}
E_{k} & =\frac{1}{2} \sum_{i}\left[m_{\mathrm{t}} W_{i}^{2}(L)+M_{i}\right] q_{i}^{\bullet 2} \\
& +\sum_{i}\left[m_{\mathrm{t}} W_{i}(L)+\int_{0}^{L} \rho A W_{i}(x) \mathrm{d} x\right] y^{\bullet} q_{i}^{\bullet} \\
& +\frac{1}{2}\left(m_{\mathrm{s}}+\rho A L+m_{\mathrm{t}}\right) y^{\bullet 2} \\
E_{\mathrm{p}} & =\frac{1}{2} \sum_{i} K_{i} q_{i}^{2}
\end{aligned}
$$

where $(\bullet)$ denotes the time derivative and $M_{i}$ and $K_{i}$ denote the $i$ th generalized mass and generalized stiffness, respectively, and can be defined as

$$
\begin{aligned}
M_{i} & =\int_{0}^{L} \rho A\left(\frac{\mathrm{d} W_{i}}{\mathrm{~d} x}\right)^{2} \mathrm{~d} x, \\
K_{i} & =\int_{0}^{L} E I\left(\frac{\mathrm{d}^{2} W_{i}}{\mathrm{~d} x^{2}}\right)^{2} \mathrm{~d} x .
\end{aligned}
$$

Equations. (6) and (7) can be substituted into following Lagrange equation (Gross et al., 2011):

$$
\frac{\mathrm{d}}{\mathrm{d} t}\left(\frac{\partial T}{\partial q_{i}^{\bullet}}\right)-\frac{\partial T}{\partial q_{i}}=Q_{i},
$$

where $T=E_{k}-E_{\mathrm{p}}$ and $Q_{i}$ represents the generalized force undergone in the flexible manipulator and satisfies the following relationship:

$$
Q_{i}=\int_{0}^{L} f(x, t) W_{i}(x) \mathrm{d} x .
$$

Here $f(x, t)$ denotes the external force generated at the clamped end $(x=0)$ of the flexible arm and can be expressed as

$$
f(0, t)=m_{\mathrm{s}} y^{\bullet \bullet}(t) .
$$

By combining Eqs. (11) and (12), the generalized force can be obtained as

$$
Q_{i}=m_{\mathrm{s}} y^{\bullet \bullet}(t) \int_{0}^{L} W_{i}(x) \mathrm{d} x .
$$

Through substitution of Eq. (13) into Eq. (10), the dynamic equation of the motor and flexible manipulator system can be obtained as

$$
\begin{aligned}
& {\left[m_{\mathrm{t}} W_{i}^{2}(L)+M_{i}\right] q_{i}^{\bullet \bullet}(t)+K_{i} q_{i}(t)} \\
& =\left[\left(m_{\mathrm{s}}-\rho A\right) \int_{0}^{L} W_{i}(x) \mathrm{d} x-m_{\mathrm{t}} W_{i}(L)\right] y^{\bullet \bullet}(t) .
\end{aligned}
$$

According to the Duhamel integral (Singiresu, 2004), the vibration response equation of the flexible arm is obtained as

$$
\begin{aligned}
q_{i}(t) & =B_{1 i} \cos \beta_{i} t+B_{2 i} \sin \beta_{i} t \\
& +\frac{\xi_{i}}{K_{i}} \int_{0}^{t} y^{\bullet \bullet}(\tau) \sin \beta_{i}(t-\tau) \mathrm{d} \tau,
\end{aligned}
$$

where 
$\beta_{i}^{2}=\frac{K_{i}}{m_{\mathrm{t}} W_{i}^{2}(L)+M_{i}}$,

$\xi_{i}=\left(m_{\mathrm{s}}-\rho A\right) \int_{0}^{L} W_{i}(x) \mathrm{d} x-m_{\mathrm{t}} W_{i}(L)$.

$B_{1 i}$ and $B_{2 i}$ are coefficients determined by the initial conditions and can be expressed as

$$
\begin{aligned}
B_{1 i} & =\frac{\rho A}{M_{i}} \int_{0}^{L} w(x, 0) W_{i}(x) \mathrm{d} x, \\
B_{2 i} & =\frac{\rho A}{M_{i} \beta_{i}} \int_{0}^{L} w^{\bullet}(x, 0) W_{i}(x) \mathrm{d} x .
\end{aligned}
$$

On the other hand, for the ball screw transmission system, the displacement of the moving slider $y(t)$ in Eq. (15) satisfies

$y^{\bullet}(t)=\frac{D}{2} \varphi^{\bullet}(t)$,

where $D$ is the diameter of the ball screw and $\phi(t)$ is the angle displacement of the electromotor shaft.

In this case, Eq. (15) further becomes

$$
\begin{aligned}
q_{i}(t) & =B_{1 i} \cos \beta_{i} t+B_{2 i} \sin \beta_{i} t \\
& +\frac{D \xi_{i}}{2 K_{i}} \int_{0}^{t} \varphi^{\bullet \bullet}(\tau) \sin \beta_{i}(t-\tau) \mathrm{d} \tau .
\end{aligned}
$$

From Eq. (20), we can obtain the result that the vibration responses of the flexible arm are related to the angle displacement of the driving motor, namely the dynamic behavior of the driving motor or the dynamic equation of the electromotor shaft should be also considered in the investigation of the dynamic analysis of the flexible manipulator.

\subsection{Vibration displacement equation of the flexible manipulator}

Section 2.1 shows that the dynamic equation of the electromotor shaft should be derived to determine the coupled dynamic equation of the system. According to the electromechanical dynamic (Wach, 2011), the dynamic equation of the electromotor shaft can be given as

$J_{\mathrm{M}} \varphi^{\bullet \bullet}(t)=M_{\mathrm{dc}}-M_{\mathrm{fz}}-M_{0}$.

Here $M_{\mathrm{dc}}$ denotes the electromagnetic torque of the electromotor relating to the types and control strategies of the motor; $M_{\mathrm{fz}}$ denotes the load torque of the electromotor, which is mainly caused by the moving slider in the slideway pair and the ball screw pair; $M_{0}$ denotes the torque loss of the electromotor caused by the power loss in the transmission system, which is much smaller than $M_{\mathrm{dc}}$; and $J_{\mathrm{M}}$ denotes the rotational inertia of the electromotor shaft, which can be expressed as

$J_{\mathrm{M}}=J_{\mathrm{R}}+J_{\mathrm{b}}+J_{\mathrm{s}}$.

Here $J_{\mathrm{R}}$ is the rotational inertia of the rotor determined by the motor type and structure, and $J_{\mathrm{b}}$ is the rotational inertia of the ball screw, which can be written as

$J_{\mathrm{b}}=\frac{\pi \rho_{\mathrm{b}} D^{4} L_{\mathrm{b}}}{32}$,

where $\rho_{\mathrm{b}}$ and $L_{\mathrm{b}}$ are the mass density and length of the ball screw, respectively.

$J_{\mathrm{S}}$ is the rotational inertia of the load which can be determined using the law of conservation of energy. The conversion rotational inertia of the load satisfies

$\frac{1}{2} J_{\mathrm{S}} \varphi^{\bullet 2}(t)=\frac{1}{2} m_{\mathrm{s}} y^{\bullet 2}(t)$.

Through combination of Eqs. (19) and (24), $J_{\mathrm{S}}$ can be obtained as

$J_{\mathrm{s}}=\frac{1}{4} m_{\mathrm{s}} D^{2}$.

To determine the dynamic equation of the electromotor shaft as shown in Eq. (21), the electromagnetic torque and the load torque of electromotor should also be determined. As indicated above, $M_{\mathrm{dc}}$ is related to the types and control strategies of the motor. In this paper, a three-phase AC motor is considered and $M_{\mathrm{dc}}$ can be expressed as

$M_{\mathrm{dc}}=\frac{P_{\mathrm{M}}}{2 \pi f_{1}} p$,

where $f_{1}$ is the power frequency, $p$ is the number of pole pairs of the motor; $P_{\mathrm{M}}$ denotes the electromagnetic power $P_{\mathrm{M}}=3 I_{2}^{\prime 2} r_{2}^{\prime} / s$, where $s$ is the slip ratio; $r_{2}^{\prime}$ is the conversion resistance of the rotor; and $I_{2}^{\prime}$ is the conversion current of the rotor, which can be further written as

$I_{2}^{\prime}=\frac{U_{\mathrm{v}}}{\sqrt{\left(r_{1}+\frac{r_{2}^{\prime}}{s}\right)^{2}+\left(x_{1}+x_{2}^{\prime}\right)^{2}}}$,

where $U_{\mathrm{v}}$ is the voltage of the motor, $r_{1}$ and $x_{1}$ are the resistance and reactance of the stator, respectively, and $x_{2}^{\prime}$ is the conversion reactance of the rotor.

Substituting Eq. (27) into Eq. (26) yields

$M_{\mathrm{dc}}=\frac{3 p}{2 \pi f_{1}} \cdot \frac{U_{\mathrm{v}}^{2} \frac{r_{2}^{\prime}}{s}}{\left(r_{1}+\frac{r_{2}^{\prime}}{s}\right)^{2}+\left(x_{1}+x_{2}^{\prime}\right)^{2}}$.

Equation (28) shows that $M_{\mathrm{dc}}$ relates to the voltage, power frequency and structure parameters of the motor. By deriving 
$M_{\mathrm{dc}}$ with respect to $s$ and assigning the result as zero, we obtain

$s_{\mathrm{m}}=\frac{r_{2}^{\prime}}{\sqrt{r_{1}^{2}+\left(x_{1}+x_{2}^{\prime}\right)^{2}}}$.

Here $s_{\mathrm{m}}$ denotes the critical slip ratio.

Through combining Eqs. (28) and (29), the maximum electromagnetic torque can be given as

$M_{\max }=\frac{3 p}{4 \pi f_{1}} \cdot \frac{U_{\mathrm{v}}^{2}}{\left[r_{1}+\sqrt{r_{1}^{2}+\left(x_{1}+x_{2}^{\prime}\right)^{2}}\right]}$.

By combining Eqs. (28) and (30), the result can be obtained that

$$
\frac{M_{\mathrm{dc}}}{M_{\max }}=\frac{2 r_{2}^{\prime}\left[r_{1}+\sqrt{r_{1}^{2}+\left(x_{1}+x_{2}^{\prime}\right)^{2}}\right]}{s\left[\left(r_{1}+\frac{r_{2}^{\prime}}{s}\right)^{2}+\left(x_{1}+x_{2}^{\prime}\right)^{2}\right]} .
$$

According to Eq. (29), Eq. (31) can be simplified as

$$
\frac{M_{\mathrm{dc}}}{M_{\max }}=\frac{2+2 \frac{r_{1}}{r_{2}^{\prime}} s_{\mathrm{m}}}{\frac{s}{s_{\mathrm{m}}}+\frac{s_{\mathrm{m}}}{s}+2 \frac{r_{1}}{r_{2}^{\prime}} s_{\mathrm{m}}} .
$$

Generally $r_{1} \approx r_{2}^{\prime}$; substituting this relation into Eq.(32) yields

$M_{\mathrm{dc}}=\frac{2 \lambda_{\mathrm{m}} M_{N}}{s_{\mathrm{m}}} s$.

Here $\lambda_{\mathrm{m}}$ denotes the overload factor of the motor and can be expressed as $\lambda_{\mathrm{m}}=M_{\max } / M_{N}, M_{N}=9550 P_{N} / n_{N}$, where $P_{N}$ and $n_{N}$ denote the rated power and the rated speed of the motor, respectively.

For the constant load torque system, $\lambda_{\mathrm{m}}$ and $s_{\mathrm{m}}$ satisfy the following relationship:

$s_{\mathrm{m}}=s_{N}\left(\lambda_{\mathrm{m}}+\sqrt{\lambda_{\mathrm{m}}^{2}-1}\right)$.

Generally $\lambda_{\mathrm{m}}=1.6 \ll 2.2$, and by combining Eqs.

and (34), $M_{\mathrm{dc}}$ can be subsequently determined.

For the MDFMS, the load torque of the electromotor is mainly generated by the moving slider, and during the motion, the friction force that the moving slider suffered in the slideway pair can be expressed as

$F_{\mathrm{s}}=\mu m_{\mathrm{s}} g$

where $\mu$ is the friction coefficient of the interface and $g=9.8 \mathrm{~m} \mathrm{~s}^{2}$.

The other part of the load torque is the friction torque generated in the ball screw pair, which can be written as

$M_{\mathrm{b}}=\frac{\mu_{0} N D}{2}$ where $\mu_{0}$ is the friction coefficient of the ball screw pair, which is relatively small for its transmitting style, and $N$ is the load on the ball screw generated by the moving slider and workpiece - this paper mainly considers that of the moving slider; thus $N=m_{\mathrm{s}} g$.

According to the law of energy conservation, we obtain

$M_{\mathrm{fz}} \varphi(t)^{\bullet} \eta_{\mathrm{t}}=F_{\mathrm{s}} y^{\bullet}(t)+M_{\mathrm{b}}$.

Here $\eta_{\mathrm{t}}$ is the energy transmission efficiency.

Through combination of Eqs. (19), (35) and (37), $M_{\mathrm{fz}}$ can be obtained as

$M_{\mathrm{fz}}=\frac{\left(\mu+\mu_{0}\right) m_{\mathrm{s}} g D}{2 \eta_{\mathrm{t}}}$.

Because the torque loss of the electromotor is relatively small, $M_{0}$ can be neglected for the purpose of simplifying the analysis.

With substitution of Eqs. (33) and (38) into Eq. (21), the dynamic equation of the electromotor shaft becomes

$J_{\mathrm{M}} \varphi^{\bullet \bullet}=\frac{2 \lambda_{\mathrm{m}} M_{N}}{s_{\mathrm{m}}} s-\frac{\left(\mu+\mu_{0}\right) m_{\mathrm{s}} g D}{2 \eta_{\mathrm{t}}}$.

Here, $s=1-n / n_{1}, n$ denotes the rotation speed of the motor, $n=60 \varphi^{\bullet} /(2 \pi), n_{1}$ is the synchronous rotation speed of the motor, and $n_{1}=60 f_{1} / p$. Thus,

$s=1-\frac{p}{2 \pi f_{1}} \varphi^{\bullet}$

Substituting Eq. (40) into Eq. (39) yields

$J_{\mathrm{M}} \varphi^{\bullet \bullet}+\frac{\lambda_{\mathrm{m}} M_{N}}{s_{\mathrm{m}}} \cdot \frac{p}{\pi f_{1}} \varphi^{\bullet}=\frac{2 \lambda_{\mathrm{m}} M_{N}}{s_{\mathrm{m}}}-\frac{\left(\mu+\mu_{0}\right) m_{\mathrm{s}} g D}{2 \eta_{\mathrm{t}}}$.

According to Eq. (41), the rotation acceleration of the motor shaft can be obtained as

$\varphi^{\bullet \bullet}=\left(\frac{2 \pi f_{1}}{p} \alpha^{2}-\alpha \gamma\right) e^{-\alpha t}$,

where $\alpha=\frac{\lambda_{\mathrm{m}} M_{N}}{J_{\mathrm{M}} s_{\mathrm{m}}} \cdot \frac{p}{\pi f_{1}}, \gamma=\frac{\left(\mu+\mu_{0}\right) m_{\mathrm{s}} g D}{2 J_{\mathrm{M}} \eta_{\mathrm{t}}}$.

With Eq. (42) substituted into Eq. (20), vibration responses of the flexible arm can be written as

$$
\begin{aligned}
q_{i}(t) & =B_{1 i} \cos \beta_{i} t+B_{2 i} \sin \beta_{i} t \\
& +\frac{D \xi_{i}}{2 K_{i}} \int_{0}^{t}\left(\frac{2 \pi f_{1}}{p} \alpha^{2}-\alpha \gamma\right) e^{-\alpha t} \sin \beta_{i}(t-\tau) \mathrm{d} \tau .
\end{aligned}
$$

With Eq. (43) substituted into Eq. (2), vibration response displacement of the flexible arm can be subsequently obtained as 


$$
\begin{aligned}
& w(x, t)=\sum_{i=1}^{n \rightarrow \infty} \\
& {\left[\begin{array}{c}
B_{1 i} \cos \beta_{i} t+B_{2 i} \sin \beta_{i} t \\
\left.+\frac{D \xi_{i}}{2 K_{i}}\left(\frac{2 \pi f_{1}}{p} \alpha^{2}-\alpha \gamma\right)\left(\begin{array}{l}
\frac{\alpha}{\alpha^{2}+\beta_{i}^{2}} \sin \beta_{i} t-\frac{\beta_{i}}{\alpha^{2}+\beta_{i}^{2}} \cos \beta_{i} t \\
+\frac{\beta_{i}}{\alpha^{2}+\beta_{i}^{2}} e^{-\alpha t}
\end{array}\right)\right] W_{i}(x),
\end{array}\right.}
\end{aligned}
$$

where

$f_{1}=\frac{p}{\pi D(1-s)} y^{\bullet}(t)$.

Equation (44) shows the vibration responses characteristic of the flexible manipulator considering the dynamic behavior of the driving motor. The situation where the flexible manipulator has velocities and accelerations is considered in this paper. For the ideal situation that the slider has constant velocities which ignore the dynamic effect of the driving motor, the velocity of the moving slider can be expressed as

$v=y^{\bullet}(t)$.

By substituting Eqs. (19), (20) and (46) into Eq. (2), the vibration response displacement of the flexible arm can be obtained as

$w(x, t)=\sum_{i=1}^{n \rightarrow \infty}\left(B_{1 i} \cos \beta_{i} t+B_{2 i} \sin \beta_{i} t\right) W_{i}(x)$.

By combining Eqs. (19), (41) and (45), $s$ in Eq. (44) can be determined as

$s_{\mathrm{v}}=\frac{\left(\mu+\mu_{0}\right) m_{\mathrm{s}} g D s_{\mathrm{m}}}{4 \lambda_{\mathrm{m}} M_{N} \eta_{\mathrm{t}}}$.

Similarly, for the ideal situation that the slider has constant accelerations, the acceleration of the moving slider can be expressed as

$a=y^{\bullet \bullet}(t)$.

With Eqs. (19), (20) and (49) substituted into Eq. (2), the vibration response displacement of the flexible arm can be obtained as

$$
\begin{aligned}
w(x, t) & =\sum_{i=1}^{n \rightarrow \infty}\left[B_{1 i} \cos \beta_{i} t+B_{2 i} \sin \beta_{i} t\right. \\
& \left.+\frac{a \xi_{i}}{\beta_{i} K_{i}}\left(1-\cos \beta_{i} t\right)\right] W_{i}(x) .
\end{aligned}
$$

By combining Eqs. (19), (41) and (49), $s$ in Eq. (44) can be determined as

$s_{\mathrm{a}}=\frac{\left(\mu+\mu_{0}\right) m_{\mathrm{s}} g D s_{\mathrm{m}}}{4 \lambda_{\mathrm{m}} M_{N} \eta_{\mathrm{t}}}+\frac{J_{\mathrm{M} s_{\mathrm{m}}}}{\lambda_{\mathrm{m}} M_{N} D} a$.

Equations (47) and (50) show the vibration responses of the flexible manipulator ignoring the dynamic effect of the driving motor. With substitution of Eqs. (48) and (51) into
Eq. (44), the vibration responses of the flexible manipulator considering the dynamic effect of the driving motor can be subsequently investigated. Through comparison investigation of Eqs. (44), (47) and (50), the dynamic effect of the driving motor on the vibration response characteristic and the effect mechanism under different motion velocities and accelerations can be investigated.

\section{Results and discussion}

In this section, numerical simulations are conducted to investigate the influence of the dynamic effect of the driving motor on the vibration responses characteristic of the flexible manipulator under different motion velocities, accelerations and structure parameters.

The parameters of the flexible arm are length $L=0.800 \mathrm{~m}$, width $b=0.080 \mathrm{~m}$, thickness $h=0.002 \mathrm{~m}$, Young's modulus $E=197 \mathrm{GPa}$ and mass density $\rho=7850 \mathrm{~kg} \mathrm{~m}^{-3}$. The driving motor is a three-phase AC motor with the parameters as follows: pole pairs $p=3$, rated power $P_{N}=2.2 \mathrm{~kW}$, rated rotation speed $n_{N}=1430 \mathrm{rmin}^{-1}$, overload factor $\lambda_{\mathrm{m}}=1.8$, and rotational inertia of the rotor $J_{\mathrm{R}}=0.0054 \mathrm{~kg} \mathrm{~m}^{-2}$. The diameter and length of the ball screw are $D=10 \mathrm{~mm}$ and $L_{\mathrm{b}}=800 \mathrm{~mm}$. The mass of the slider $m_{\mathrm{s}}=500 \mathrm{~g}$, and the mass of the end effector $m_{\mathrm{t}}=50 \mathrm{~g}$. The energy transmission efficiency $\eta_{\mathrm{t}}=0.9$, the friction coefficient of the slideway $\mu=0.004$, and the friction coefficient of the ball screw $\mu_{0}=0.001$.

Figure 2 shows the vibration responses of the MDFMS under velocity motions and acceleration motions. During the simulations, the motion velocity and acceleration are assigned as $v=0.05 \mathrm{~m} \mathrm{~s}^{-1}$ and $a=1.0 \mathrm{~m} \mathrm{~s}^{-2}$. It can be seen that the vibration responses considering the dynamic effect of the driving motor are different to the ideal situation ignoring the dynamic effect of the driving motor. The response amplitudes are larger than those of the ideal situation, especially in the initial time, for the acceleration motions. This indicates that the dynamic effect of the driving motor increases the vibration responses and the ideal situation will cause an error in the dynamic analysis of the flexible manipulator.

Figure 3 shows the frequency responses of the MDFMS under velocity motions. During the simulations, the motion velocity is assigned as $v=0.05 \mathrm{~m} \mathrm{~s}^{-1}$. The result indicates that the frequency responses considering the dynamic effect of the driving motor are similar to the ideal situation. It should be noted that a consistent result can also be obtained for the MDFMS that has acceleration motions. Figures 2 and 3 provide a conclusion that the dynamic effect of the driving motor has a significant influence on the dynamic characteristic of the flexible manipulator, primarily in that it increases the vibration response amplitude.

To further investigate the effect mechanism of the driving motor on the vibration responses, vibration responses of the MDFMS under different motion velocities and accelerations 

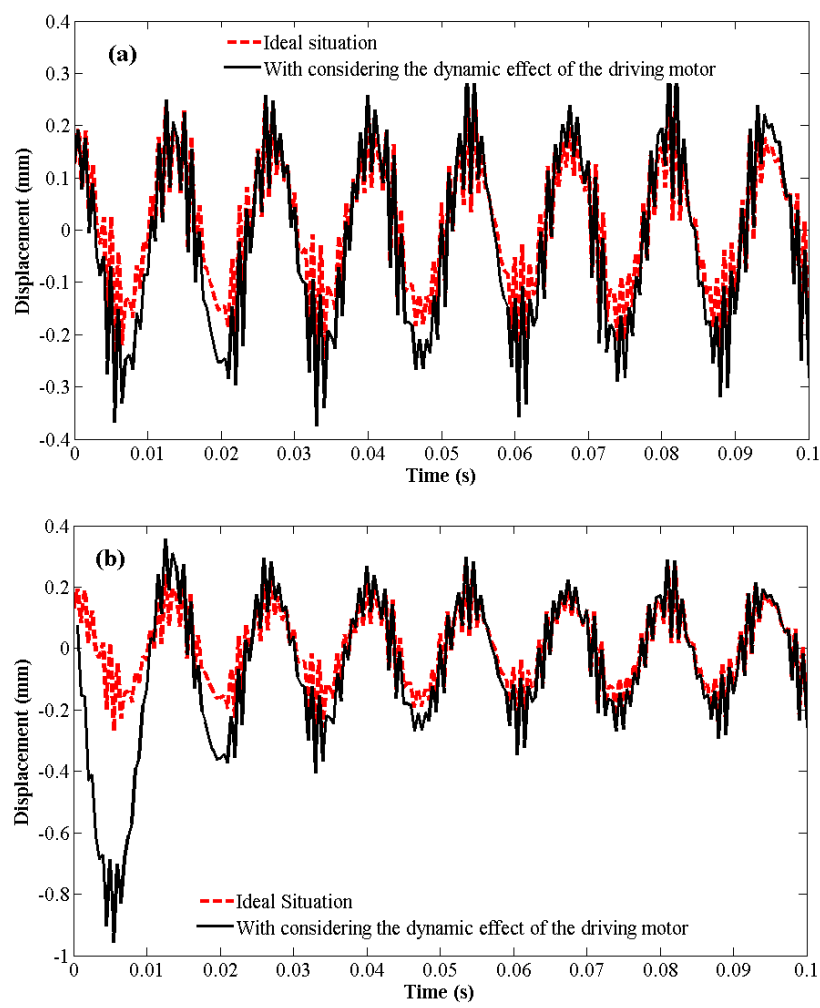

Figure 2. Vibration responses of the MDFMS under velocity (a) and acceleration (b) motions.

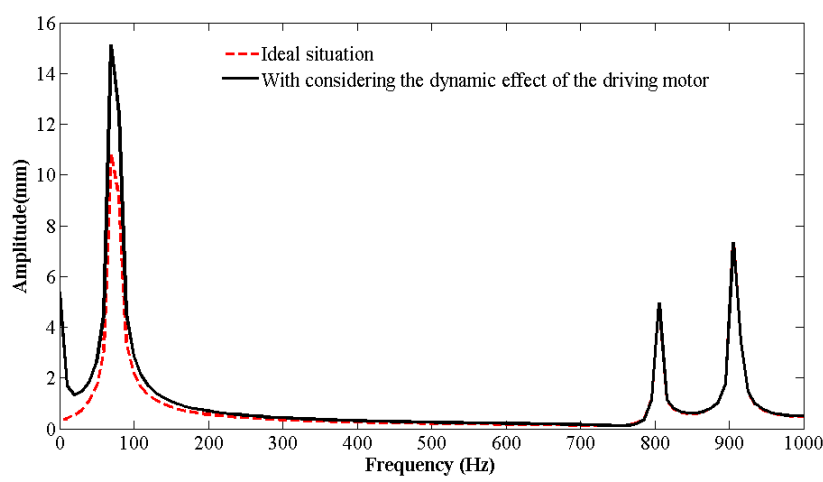

Figure 3. Frequency responses of the MDFMS under velocity motions.

are presented in Figs. 4 and 5. With the purpose of simplifying the analysis process, only the results of steady-state responses are considered in the following investigations.

Figure 4 shows the vibration responses of the MDFMS under different motion velocities. During the simulations, the motion velocities are assigned as $v=0.05,0.1$ and $0.15 \mathrm{~m} \mathrm{~s}^{-1}$. It can be seen that, for the ideal situation ignoring the dynamic effect of the driving motor, the steadystate responses of different motion velocities are consistent and show minor changes, as described by the dotted red line, which can also be obtained from Eq. (47). However, consid-

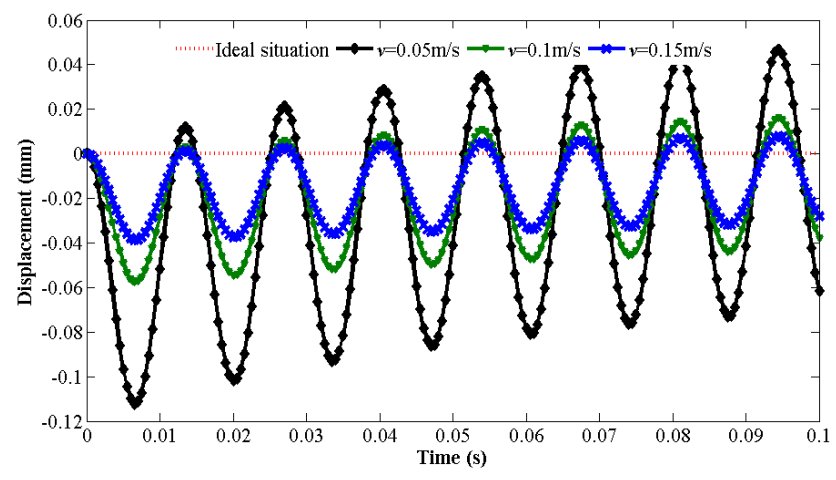

Figure 4. Vibration responses of the MDFMS under different motion velocities.

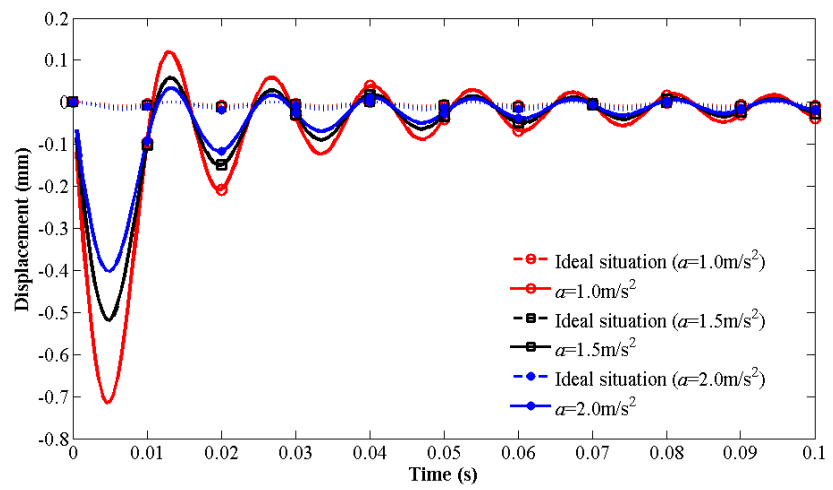

Figure 5. Vibration responses of the MDFMS under different motion accelerations.

ering the dynamic effect of the driving motor, the velocities exhibit noticeable influence on the vibration responses and the response amplitudes are larger than the ideal situations; moreover, as the velocities increase, the response amplitudes become smaller and the influence decreases.

The vibration responses of the MDFMS under different motion accelerations are presented in Fig. 5. During the simulations, the motion accelerations are assigned as $a=1.0$, 1.5 and $2.0 \mathrm{~m} \mathrm{~s}^{-2}$. It can be found that the vibration responses considering the dynamic effect of the driving motor are obviously larger than the ideal situation, and the results show an obvious difference, especially in the initial time; as time progresses, the difference decreases and the results gradually approach the ideal situation. Moreover, if we do not consider the dynamic effect of the driving motor, the influence of accelerations on the vibration responses is not obvious. However, when the dynamic effect of the driving motor is considered, the accelerations show a considerable influence; as the accelerations increase, the difference of the results and the influence of the driving motor decrease, which is similar to the velocity motion situation. 

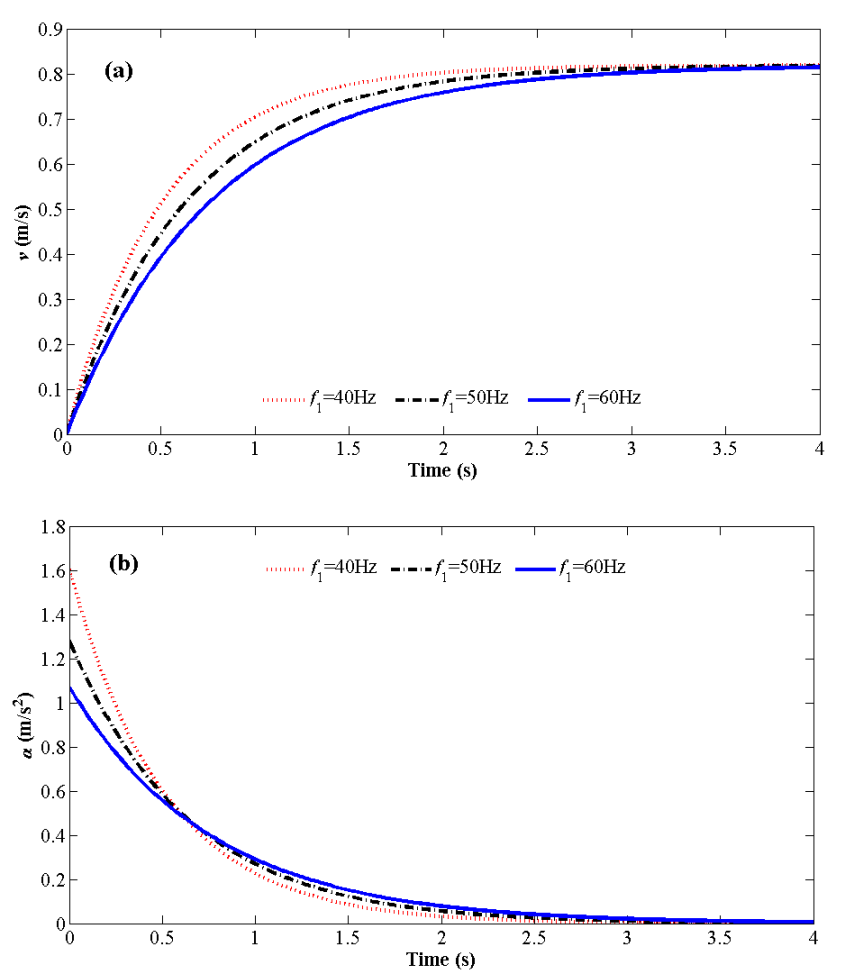

Figure 6. Velocity (a) and acceleration (b) characteristics of the MDFMS under varied power frequencies.

Figures 4 and 5 demonstrate that the influence of the dynamic effect of the driving motor is more considerable, especially in the initial time, and the influence decreases as the velocities and accelerations increase, which indicates that larger velocities and accelerations have a suppressing effect on the influence of the driving motor. As we know, for a constant moment load, the velocity and acceleration characteristics and the response time of the system are determined by the power frequency. Therefore, to illustrate this phenomenon, Fig. 6 shows the velocity and acceleration characteristics of the MDFMS under different power frequencies assigned as $f_{1}=40,50$ and $60 \mathrm{~Hz}$. We can obtain the result that, for lower power frequencies, the velocity response and the acceleration response are more rapid, and the vibration responses are more intense, as shown in Fig. 7, which can also be obtained from Eq. (28,) which gives a description that lower power frequencies can obtain larger electromagnetic torques; as a result, if the load torques are constant, the response processes will become more rapid.

The flexible arm and the end effector are the main components of the operation system; both have a significant influence on the structural service and dynamic performance. Generally, the flexible arm can be structured using lightweight materials of different Young's modulus, and the end effector can be established with different masses. Figure 8 shows the vibration responses of the flexible arm with a different Young's modulus, assigning $E=65$,

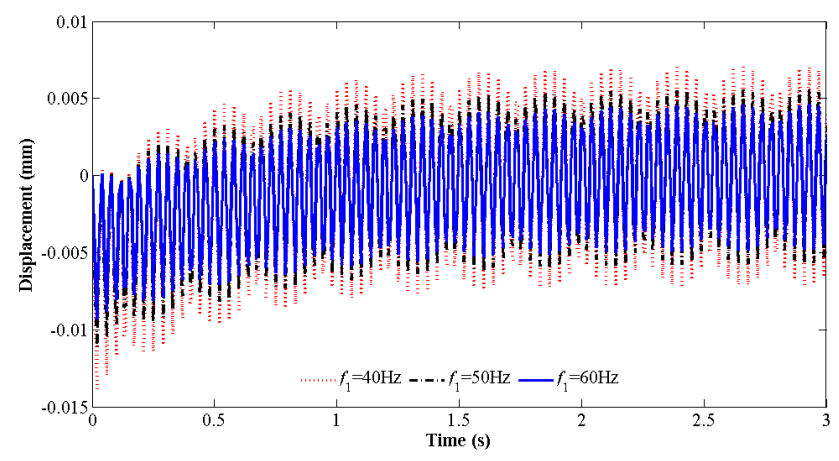

Figure 7. Vibration responses of the MDFMS under varied power frequencies.
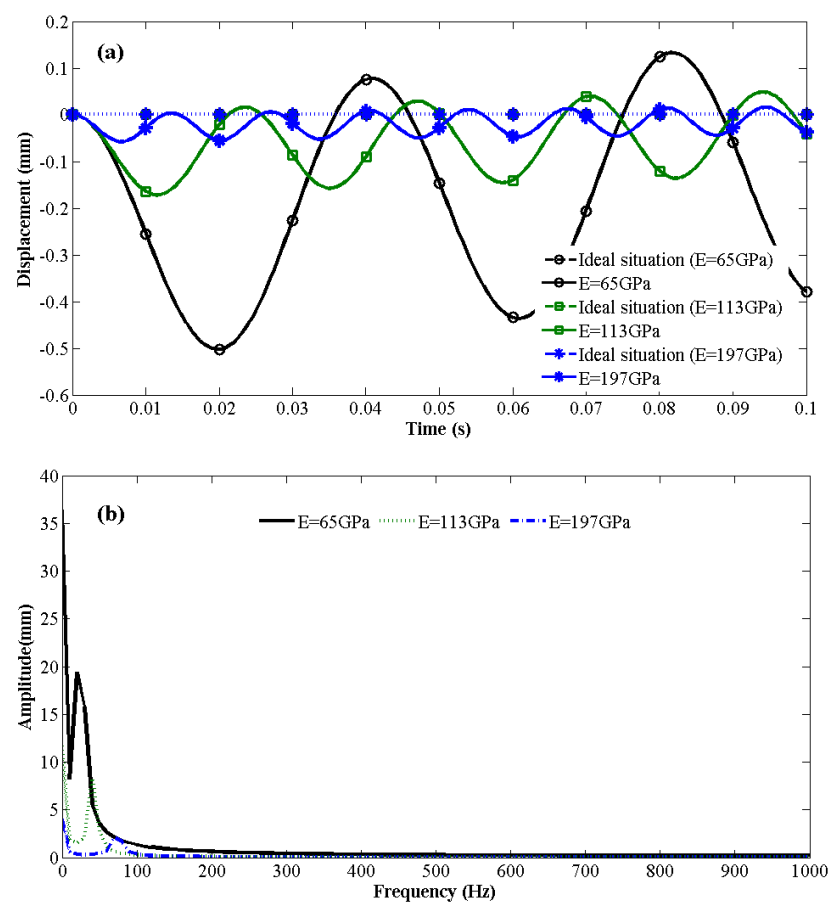

Figure 8. Effect of Young's modulus on the vibration responses of the MDFMS: (a) response amplitudes and (b) response frequencies.

113 and $197 \mathrm{GPa}$. The result reveals that Young's modulus will change the dynamic performance and have considerable influence on the vibration responses. If the flexible arm is structured using a smaller Young's modulus, the vibration response amplitudes increase and the response frequencies become lower, which indicates that the vibration responses are enhanced and lower-frequency vibrations are easily excited. Moreover, in this case the influence of the driving motor will become more noticeable and should be given more consideration.

Figure 9 shows the influence of the end effector on the vibration responses. During the simulations, the mass of the end effector is assigned as $m_{\mathrm{t}}=10,20$ and $50 \mathrm{~g}$. We can see that the vibration responses vary with the mass of the end 

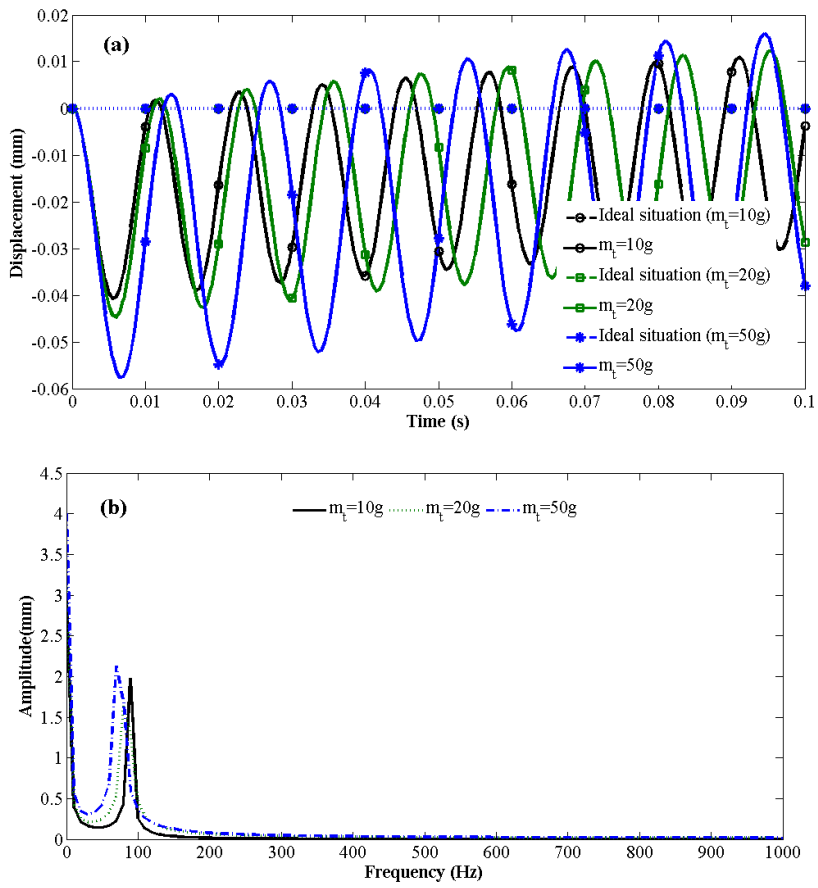

Figure 9. Effect of the end effector on the vibration responses of the MDFMS: (a) response amplitudes and (b) response frequencies.

effector; for lighter end effectors, the response amplitudes are smaller, and as mass increases, the response amplitudes increase while the response frequencies decrease. This result can also be obtained from Eq. (16). This indicates that a lightweight end effector contributes to suppressing the vibration responses of the arm, which is meaningful for the structure design and vibration control of the flexible manipulator.

\section{Conclusions}

A coupled dynamic model of the MDFMS is established which can clearly reflect the dynamic effect of the driving motor. Based on the proposed coupled dynamic model, the vibration responses of the flexible manipulator under different velocities, accelerations and structure parameters, as well as the effect mechanism of the driving motor on the vibration responses, are investigated. The results demonstrate that the dynamic effect of the driving motor has a significant influence on the dynamic characteristic of the flexible manipulator, primarily in that it increases the vibration responses amplitude; furthermore, the ideal assumption ignoring the dynamic effect of the driving motor, which is generally regarded in recent research, will cause an error in the dynamic analysis, especially in the initial time. The response amplitudes are larger than the ideal situations; as the velocities and accelerations increase, the response amplitudes become smaller and the influence decreases. It can also be observed that larger velocities and accelerations have a suppressing effect on the influence of the driving motor. Moreover, the
Young's modulus of the flexible arm and the mass of the end effector have a considerable effect on the vibration responses, which indicates that a smaller Young's modulus increases the vibration response amplitudes and the influence of the driving motor is more noticeable; further, a lightweight end effector contributes to suppressing the influence of the driving motor. The results obtained in this paper are meaningful for the structure design, motion optimization and dynamic analysis of the flexible manipulator.

Acknowledgements. This research work is supported by the National Natural Science Foundation of China (no. 51305444), the Doctoral Fund of Ministry of Education (no. 20120095120013), the Scientific and Technological Project of Jiangsu Province (BY2014028-06), and the project funded by the Priority Academic Program Development of Jiangsu Higher Education Institutions (PAPD). The authors sincerely thank the reviewers for their significant and constructive comments and suggestions, which substantially helped in improving this paper.

Edited by: K. Mianowski

Reviewed by: R. Sato and another anonymous referee

\section{References}

Andreaus, U. and Casini, P.: Dynamics of friction oscillators excited by a moving base and/or driving force, J. Sound Vibrat., 245, 685-699, 2001.

Dwivedy, S. K. and Eberhard, P.: Dynamic analysis of flexible manipulators, a literature review, Mech. Mach. Theory, 41, 749777, 2006.

Ge, S. S., Lee, T. H., and Zhu, G.: Asymptotically stable end-point regulation of a flexible SCARA/Cartesian robot, IEEE/ASME T. Mechatron., 3, 138-144, 1998.

Gross, D., Hauger, W., Schröder, J., Wall, W. A., and Govindjee, S. Engineering Mechanics 3: Dynamics, Springer, London, 2011.

Kerem, G., Bradley, J. B., and Edward, J. P.: Vibration control of a single-link flexible manipulator using an array of fiber optic curvature sensors and PZT actuators, Mechatronics, 19, 167-177, 2009.

Li, Z. J., Cai, G. W., Huang, Q. B., and Liu, S. Q.: Analysis of nonlinear vibration of a motor-linkage mechanism system with composite links, J. Sound Vibrat., 311, 924-940, 2008.

Liou, F. W., Erdman, A. G., and Lin, C. S.: Dynamic analysis of a motor-gear-mechanism system, Mech. Mach. Theory, 26, 239252, 1991.

Liu, Y. F., Li, W., Yang, X. F., Fan, M. B., Wang, Y. Q., and Lu, E.: Vibration response and power flow characteristics of a flexible manipulator with a moving base, Shock and Vibration, Hindawi Publishing Corporation, New York, 1-8, 2015.

Maria, A. N., Jorge, A. C., Ambrósio, L. M., Roseiro, A. A., and Vasques, C. M. A.: Active vibration control of spatial flexible multibody systems, Multibody Sys. Dynam., 30, 13-35, 2013.

Mohsen, D., Nader, J., Zeyu, L., and Darren, M D.: An observerbased piezoelectric control of flexible cartesian robot arms: theory and experiment, Control Eng. Pract., 12, 1041-1053, 2004. 
Qiu, Z. C.: Adaptive nonlinear vibration control of a Cartesian flexible manipulator driven by a ball screw mechanism, Mech. Syst. Signal Proc., 30, 248-266, 2012.

Qiu, Z. C., Shi, M. L., Wang, B., and Xie, Z. W.: Genetic algorithm based active vibration control for a moving flexible smart beam driven by a pneumatic rod cylinder, J. Sound Vibrat., 331, 2233 2256, 2012.

Siciliano, B. and Khatib, O.: Springer Handbook of robotics, Springer, London, 2008.

Singiresu, S. R.: Mechanical Vibration, 4th Edn., Pearson Education Inc., New York, 2004.

Smaili, A., Kopparapu, M. and Sannah, M.: Elastodynamic response of a d.c motor driven flexible mechanism system with compliant drive train components during start-up, Mech. Mach. Theory, 31, 659-672, 1996.

Tuttle, T. D. and Seering, W. P.: A nonlinear model of a harmonic drive gear transmission, IEEE T. Robot. Automat., 12, 368-374, 1996.
Wach, P.: Dynamics and Control of Electrical Drives, Springer, London, 2011.

Wei, K. X., Meng, G., Zhou, S., and Liu, J. W.: Vibration control of variable speed/acceleration rotating beams using smart materials, J. Sound Vibrat., 298, 1150-1158, 2006.

Zhang, X., Mills, J. K., and Cleghorn, W. L.: Investigation of axial forces on dynamic properties of a flexible 3-PRR planar parallel manipulator moving with high speed, Robotica, 28, 607-619, 2010.

Zhao, J. L., Yan, S. Z., and Wu, J. N.: Analysis of parameter sensitivity of space manipulator with harmonic drive based on the revised response surface method, Acta Astronaut., 98, 86-96, 2014. 